\title{
DYNAMICAL EVOLUTION OF SPECTRAL AND TIMING PROPERTIES OF COMPACT OBJECTS: SOME EXAMPLES
}

\author{
SANDIP K. CHAKRABARTI* \\ S. N. Bose National Centre for Basic Sciences, \\ JD Block, Salt Lake, Kolkata 700098, India \\ chakraba@bose.res.in \\ PARTHA S. PAL \\ Indian Centre for Space Physics, 43 Chalantika, \\ Garia Station Rd., Kolkata 700084, partha@csp.res.in
}

\begin{abstract}
We present examples of rapid changes in spectral and timing properties in accretion flows around compact objects and discuss what could be going on in these systems. We find a new way of quantifying the variation of the flow geometry. We show the evolution of the variation of the Comptonization efficiency computed from the ratio of the Comptonized photons and the injected seed photons. The time evolution is a direct consequence of the variation of the accretion rates which changes the hydrodynamic and radiative properties of the flow and therefore the flow geometry.
\end{abstract}

Keywords: Black hole physics, radiative transfer, accretion disks, outbursts, gamma ray bursts

\section{Introduction}

Accretion flows around compact objects may evolve very rapidly since the time scales $R_{g} / c$ could vary from few tens of microseconds for a stellar mass $M \sim M_{\odot}$ to few tens of thousands of seconds for a super-massive black hole $M \sim$ a few $\times$ $10^{9} M_{\odot}$, where $R_{g}$ is the length scale $\sim 2 G M / c^{2}$ of the system and $c$ is the velocity of light. In reality, the observational constraints (distance, errors, poor statistics, instrumental limitations) do not enable us to study variabilities at such a short timescales especially for stellar mass black holes. Even then, timescale of variabilities seen are sufficiently small, say few milliseconds to a few seconds. This posits a big challenge to the theoreticians.

As we are aware of, the black holes are black and thus they must be observed by detecting the radiations emitted by accreting matter. A short time scale variations in accreting systems naturally lead to suspicion that the object might be compact. Over

*Also at Indian Centre for Space Physics, 43 Chalantika, Garia Station Rd., Kolkata 700084 
the last decade, several satellites such as Beppo SAX, RXTE, INTEGRAL, XMMNEWTON and most recently SWIFT, FERMI etc. brought huge amount of data from compact systems such as Gamma Ray Bursts (GRBs), Black holes and neutron stars. These data showed evolutions of both the spectral and timing properties in a very short time scale. In this article, we shall concentrate on the stellar mass black hole GRS $1915+105$ and show that both the radiation spectrum and the power density spectrum are very dynamic. The spectrum generally contains a soft component (supposed to be arising out of the Keplerian disk; Shakura \& Sunyaev, 1973) and a power-law component (supposed to be arising out of Comptonization of some of the soft photons by a so called hot electron cloud; Sunyaev \& Titarchuk, 1980; Coppi \& Zdziarski, 1992; Haardt \& Maraschi, 1993; Chakrabarti \& Titarchuk, 1995; Kobayashi et al. 2003). One of the ways to quantify the dynamism is to study the ratio of the number of photons participating in the Comptonization process vis-a-vis the number of injected soft photons (we can call this as Comptonizing efficiency CE). This should give some information related to the geometry of the so-called Compton cloud, since the number of power-law photons is proportional to those which are actually intercepted by the Compton cloud. All of the intercepted photons may or may not actually be Comptonized as that would depend on the optical depth of the cloud. Independent of whether soft photons are arising out of a Keplerian disk or some other optically thick medium or shell, nevertheless, CE appears to be a useful parameter to see how the geometry of the Compton cloud changes with time. Other than GRS1915+105 we shall discuss a couple of outburst sources, XTE 1550-564 and GX 339-4 and a GRB source GRB090618 which Indian payloads RT-2 observed while on board CORONAS-PHOTON (Rao et al. 2011).

\section{Time variabilities of the Black Hole candidate GRS $1915+105$}

Continuous X-ray observation of GRS $1915+105$ reveals that the X-ray intensity of the source changes peculiarly in a variety of timescales ranging from seconds to days (Greiner et al., 1996, Morgan et al., 1997). Quasi-Periodic Oscillations (QPOs) are observed in a wide range of frequencies. QPOs in this source are associated with different types of X-ray variabilities and their timing properties are correlated with spectral features (Muno et al., 1999). The origin of QPO frequencies between 0.5 to $\sim 20 \mathrm{~Hz}$ is identified to be due to the oscillation of the Comptonized photons, emitted from the post-shock region (centrifugal barrier) of the low angular momentum (sub-Keplerian) flow (Chakrabarti \& Manickam, 2000, hereafter CM00; Rao et al., 2000). Small scale variabilities of GRS $1915+105$ were thought to be due to with local variation of the inner disk (Nandi et al., 2000, Migliari \& Belloni, 2003). Several observers have reported that this object exhibited many types of variability classes (Yadav et al., 1999; Rao, Yadav \& Paul, 2000; Belloni et al. 2000, Chakrabarti \& Nandi, 2000; Naik et al. 2002a). Depending on the variation of photon counts in different arbitrary energy bands (hardness ratio) and color-color diagram of GRS $1915+105$, the X-ray variability of the source was found to have fifteen arbitrarily 
named $\left(\alpha, \beta, \gamma, \delta, \phi, \chi_{1}, \chi_{2}, \chi_{3}, \chi_{4}, \mu, \nu, \lambda, \kappa, \rho, \theta\right)$. In 1999 observation of RXTE, existence of another class $\omega$ was reported (Klein-Wolt et al., 2002; Naik et al. $2002 \mathrm{a}$ ). In the so-called $\chi$ (i.e., $\chi_{1}$ to $\chi_{4}$ ) class, the strong variability as is found in other classes is absent. The classes named $\chi_{1}, \chi_{3}, \beta$ and $\theta$ are associated with the presence of strong radio jets (Naik \& Rao, 2000, Vadawale et al., 2003).

In the last decade, based on the Indian X-ray Astronomy Experiment (IXAE) on board IRS-P3 (Chakrabarti et al. 2004 and 2005) pointed out that switching from one variability class (usually through an intermediate class) to another actually takes a couple of hours justifying the assumption that the low angular momentum flow plays a major role. Pal et al. (2011ab) pointed out that different the variability classes actually have different Comptonization efficiencies, independent of the model of the Compton cloud, and concluded that the average geometry of the disk must be changed from one variability to another and not only that, within a single class, the variation of spectral states from burst-on to burst-off would require the geometry to change in a matter of few seconds.

To show this we consider one set of the RXTE science data from the NASA HEASARC data archive and analyzed them. We wish to compute the Comptonization efficiency of all the sixteen variability classes renamed as $\mathrm{I}=\phi ; \mathrm{II}=\delta ; \mathrm{III}=\gamma$; $\mathrm{IV}=\omega ; \mathrm{V}=\mu ; \mathrm{VI}=\nu ; \mathrm{VII}=\lambda ; \mathrm{VIII}=\kappa ; \mathrm{IX}=\rho ; \mathrm{X}=\beta ; \mathrm{XI}=\alpha ; \mathrm{XII}=\theta ; \mathrm{XIII}=\chi_{2} ; \mathrm{XIV}=\chi_{4}$; $\mathrm{XV}=\chi_{1} ; \mathrm{XVI}=\chi_{3}$; and sequence them in an increasing or decreasing order. This resolves a long standing problem: What is the sequence in which the sixteen classes appear? In fact, the observed transitions actually correspond to a transition from one class to the next one in this sequence (Pal, Chakrabarti and Nandi, 2011b).

In Table. 1, the details of the data selection and ObsIDs are given which we analysed in this paper. The Data set having a 'star' mark are analyzed and presented in Fig. 3, while the CE CE of both the sets of data are in Fig. 4.

\section{Computation of the Comptonizing Efficiency}

Since we are interested in computing the ratio of the power-law photons and the injected soft photons, We need to fit the spectrum at each instant with a black body and a power law. Spectral analysis for the PCA data is done by using "standard2" mode data which have $16 \mathrm{sec}$ time resolution and we constrained our energy selection up to $40 \mathrm{keV}$ to match with the timing analysis. The source spectrum is generated using FTOOLS task "SAEXTRCT" with 16 sec time bin from "standard2" data. The background fits file is generated from the "standard2" fits file by the FTOOLS task "runpcabackest" with the standard FILTER file provided with the package. The background source spectrum is generated using FTOOLS task "SAEXTRCT" with 16 sec time bin from background fits file. The standard FTOOLS task "pcarsp" is used to generate the response file with appropriate detector information. The spectral analysis and modeling was performed using XSPEC (v.12) astrophysical fitting package. For the model fitting of PCA spectra, we have used a systematic error of $1 \%$. The spectra are fitted with diskbb and power law model along with 


\begin{tabular}{ccc} 
Obs-Id & Class & Date \\
\hline \hline $10408-01-09-00^{*}$ & I & $29-05-1996$ \\
$10408-01-19-00$ & I & $29-06-1996$ \\
$10408-01-18-00^{*}$ & II & $25-06-1996$ \\
$20402-01-41-00$ & II & $19-08-1997$ \\
$20402-01-37-00^{*}$ & III & $17-07-1997$ \\
$20402-01-56-00$ & III & $22-11-1997$ \\
$40703-01-27-00^{*}$ & IV & $23-08-1999$ \\
$40403-01-07-00$ & IV & $23-04-1999$ \\
$10408-01-36-00^{*}$ & V & $28-09-1996$ \\
$20402-01-53-01$ & V & $05-11-1997$ \\
$10408-01-40-00^{*}$ & VI & $13-10-1996$ \\
$20402-01-02-02$ & VI & $14-11-1996$ \\
$20402-01-36-00 *$ & VII & $10-07-1997$ \\
$10408-01-38-00$ & VII & $07-10-1996$ \\
$20402-01-35-0 *^{*}$ & VIII & $07-07-1997$ \\
$20402-01-33-00$ & VIII & $18-06-1997$ \\
$20402-01-31-00^{*}$ & IX & $03-06-1997$ \\
$20402-01-03-00$ & IX & $19-11-1996$ \\
$10408-01-10-00^{*}$ & X & $26-05-1996$ \\
$20402-01-44-00$ & X & $31-08-1997$ \\
$20187-02-01-00^{*}$ & XI & $07-05-1997$ \\
$20402-01-30-01$ & XI & $28-05-1997$ \\
$10408-01-15-00^{*}$ & XII & $16-06-1996$ \\
$20402-01-45-02$ & XII & $05-09-1997$ \\
$20402-01-16-00^{*}$ & XIII & $22-02-1997$ \\
$20402-01-05-00$ & XIII & $04-12-1996$ \\
$20402-01-25-00 *$ & XIV & $19-04-1997$ \\
$10408-01-33-00$ & XIV & $07-09-1996$ \\
$10408-01-23-00^{*}$ & XV & $14-07-1996$ \\
$10408-01-30-00$ & XV & $18-08-1996$ \\
$20402-01-50-00^{*}$ & XVI & $14-10-1997$ \\
XI-01-51-00 & XVI & $22-10-1997$
\end{tabular}

$6.0 \times 10^{22} \mathrm{~cm}^{-2}$ hydrogen column absorption (Muno et al., 1999) and we used the Gaussian for iron line as required for best fitting. To have the spectral evolution with time for each class, we have generated PCA spectrum (2 to 40keV) with a minimum of $16 \mathrm{~s}$ time interval along with background spectrum and response matrix. This procedure is repeated with every $16 \mathrm{~s}$ shift in time interval since the minimum time resolution in 'standard2' data is 16s. Details are in Pal, Chakrabarti and Nandi (2011a). 
In Fig. 1, we show an example of the fitted PCA and HEXTE spectrum along with the fitted components. We also show the residuals to characterize the goodness of the fit. In the left panel, the fitting is done with diskbb and power-law components. In the right panel, the same spectrum is fitted with diskbb and compST models. The photon numbers and CE are calculated with the parameters obtained from the fitting. The black body spectrum is simulated with $T_{i n}=1.15_{-0.052}^{+0.057} \mathrm{keV}$. The calculated number of black body photons from $0.1-6.5 \mathrm{keV}$ is $156.03_{-13.90}^{+17.10}$ kcounts/s. In the same way, the power-law spectrum is simulated with the power-law index $=2.057_{-0.147}^{+0.154}$. The calculated number of the Comptonized photons between $3.45-40 \mathrm{keV}$ is $0.39{ }_{-0.05}^{+0.06} \mathrm{kcounts} / \mathrm{s}$. The ratio between the power-law photon and the black body photon is $0.25{ }_{-0.07}^{+0.08} \%$. This means that only $0.41 \%$ of the soft photons are Comptonized by the CENBOL. For the sake of comparison, the simulation with the compST model parameters are found to be $K T_{e}=6.41_{-1.25}^{+2.55}$ and $\tau=9.42_{-2.39}^{+2.88}$. The number of Comptonized photons within the same range as previous case is $0.41_{-0.05}^{+0.06} \mathrm{kcounts} / \mathrm{s}$. Thus the ratio between the compST photon and the diskbb photon is $0.26{ }_{-0.07}^{+0.08 \%}$. In Fig. 1a, we also include HEXTE data, and fitted with a power-law. But the spectral fit does not change in slope and the number of photons contributed by HEXTE regime is so low that the basic result of $\mathrm{CE}$ is not changed. In Fig. 1b, we give an example where comptST model cannot be fitted with HEXTE data. This is true for many variability classes. We therefore ignore the HEXTE data altogether. One sample spectrum is taken for each class and fitted with the diskbb and compST model with $n_{H}=6.0 \times 10^{22} \mathrm{~cm}^{-2}$ hydrogen column for absorption and $1 \%$ systematic error.

We present the dynamical analysis one by one. The Observational IDs are given in Table 1. Though CE computed as above did not depend on models, it may be easy to use a model for visualization. Assuming generalization of the Chakrabarti \& Titarchuk (1995) model, we draw in Fig. 2(a-c), a cartoon diagram where three possible outcomes of the two component advective flow (TCAF) model are shown. In Fig. 2a, where the spectrum is hard, we expect a large CE, as the soft photons from the Keplerian flow are intercepted by the CENBOL and outflow. Soft photons are not high enough to cool the Compton cloud. In Fig. 2b, where the spectrum is soft, CE should be very small. Here the Compton cloud is totally cooled by the Keplerian disk. In Fig. 2c, the spectrum may be time dependent due to interactions between the outflow and the radiation and the CE should vary according to whether the object is in burst-on or burst-off state (Chakrabarti \& Manickam, 2000).

We now present the dynamical analysis of the light curves of most of the variability classes (Figs. 3a-3p). Each Figure contains four panels. The top panel is the variation of the photon counts with time (light curve). The second panel is the variation of $\log$ (power) of the dynamical Power Density Spectra (PDS) which may show presence or absence of quasi-periodic variations or QPOs. The third panel is the dynamic energy spectrum which shows whether the variability class is dominated by hard photons or soft photons. Finally, the bottom panel shows the variation of 

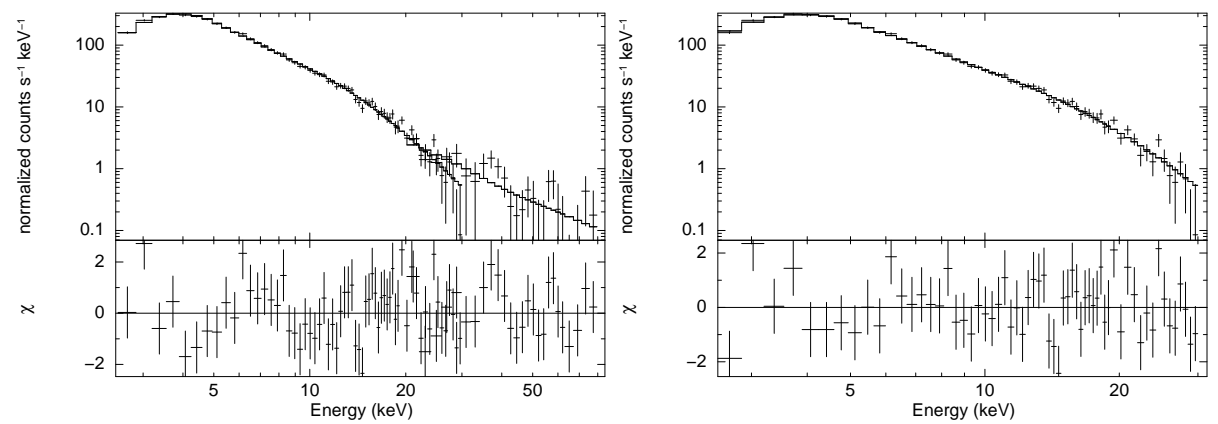

Fig. 1. A sample fitted PCA spectrum along with the diskbb and power law component (left). The HEXTE data is also added. The same PCA spectrum is fitted with diskbb and compST components (right).

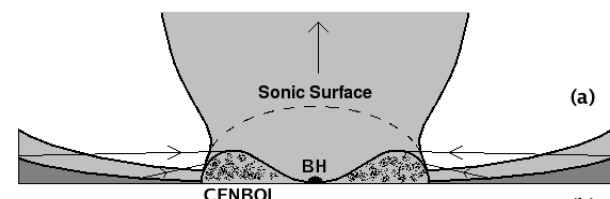

(b)
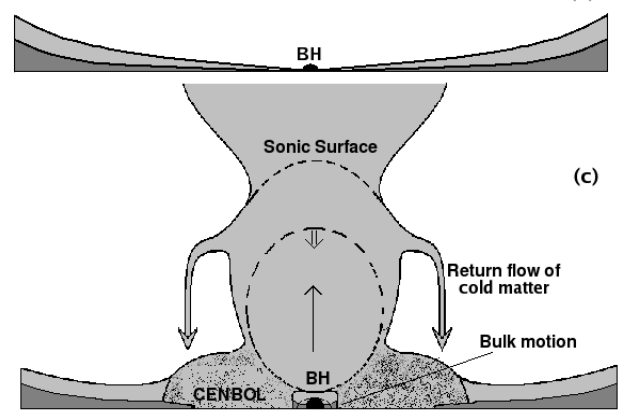

Fig. 2. Cartoon diagrams for three major types of variabilities in GRS 1915+105. It appears that the classes XIII-XVI belong to the group (a) where the Keplerian rate is low, the CENBOL is large and $\langle C E\rangle$ is high. Jet/outflow rates may be low and continuous. The softer classes such as I-III belong to group (b) where the CENBOL is very small and very little Comptonization may be due to the sub-Keplerian flow only. The rest of the classes belong to variations of group (c), where, the jet also plays a dynamically important role in shaping the spectrum.

Comptonizing Efficiency CE calculated using 16 seconds of binned data. The error bar is provided at $90 \%$ confidence level.

From these figures we see that both the radiation spectrum and the power density spectrum are highly time dependent. The spectral transition from the burst-on to burst-off states and vice versa are clearly observed within many of the classes and at the same time the higher power is observed in certain frequency range when the object is in spectrally harder state.

The variation of $\mathrm{CE}$ in the bottom panel also clearly show that during spectrally harder states (burst-off) and in particular in spectrally harder classes (e.g., $\chi$ ), the 
$\mathrm{CE}$ is larger as compared to that in the spectral softer (burst-on) classes. CE was not seen to follow the light curves exactly because the binsize of $16 \mathrm{~s}$ used to compute CE is too large. In Fig. 4 we arrange the $\mathrm{CE}$ of all the sixteen classes of two independent sets of RXTE data of GRS 1915+105 with filled squares and filled triangles. They behave almost identically.
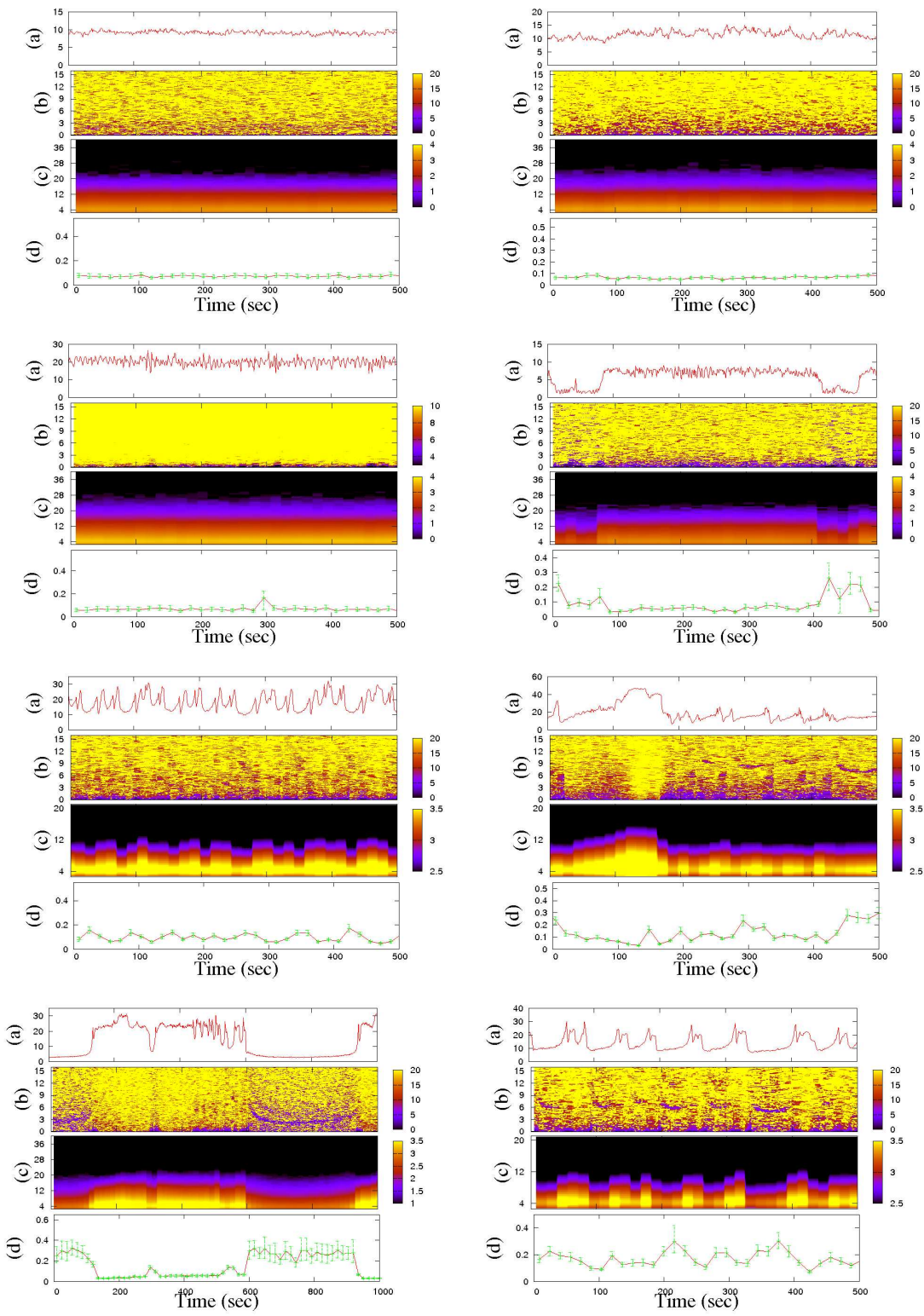

Fig. 3: Continued 

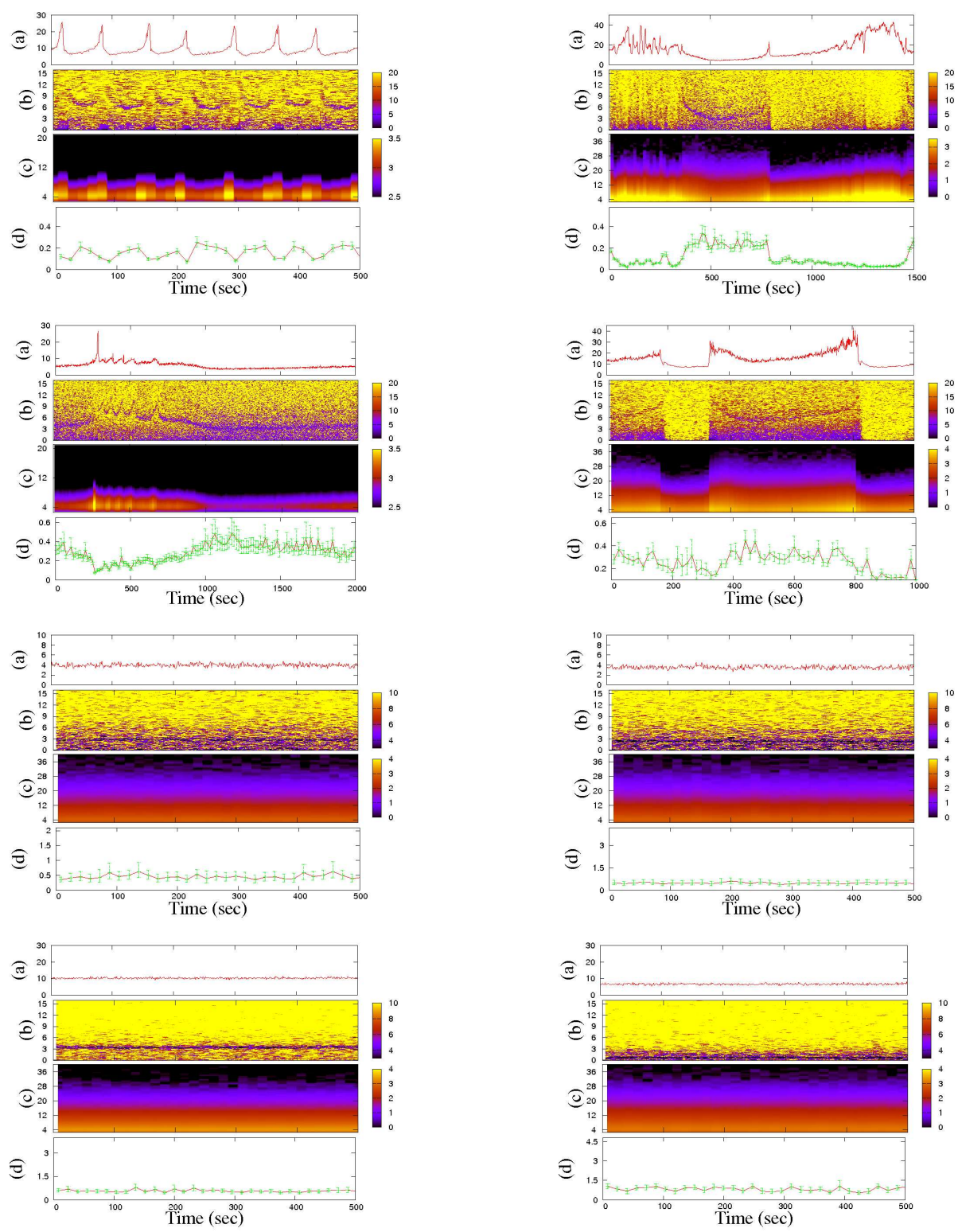

Fig. 3. Results of the analysis of (a) I (top left), (b) II (top right), (c) III (2nd row left), (d) IV (2nd row right etc.), (e) V, (f) VI, (g) VII, (h) VIII, (i) IX, (j) X, (k) XI, (l) XII, (m) XIII, (n) XIV, (o) XV and (p) XVI classes are shown. First Panel: Light curve in 2.0-40.0 keV range, Middle Panel: Dynamic PDS of the light curve. Clear evidence of low frequency noise and QPOs are seen. Third panel: Dynamic PCA spectrum showing subtle variations of the spectral characteristics with time. Bottom Panel: Comptonizing Efficiency (CE) in percent, obtained from 16s binned data. 


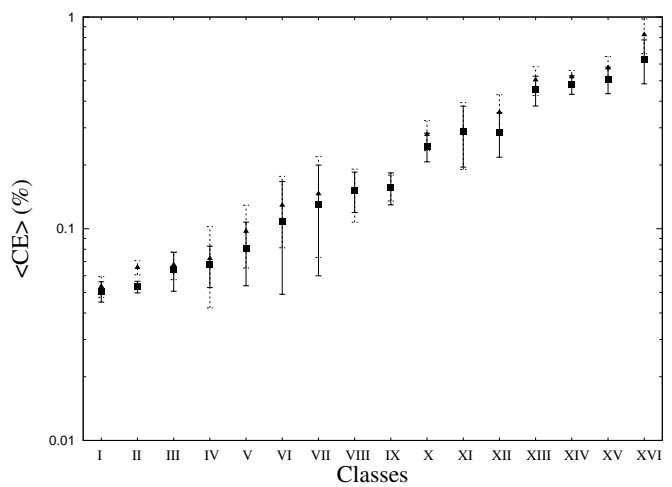

Fig. 4. Variation of average Comptonizing efficiency $(\langle C E\rangle)$ for different variability classes of GRS 1915+105. The error-bars were calculated from the excursion of CE in a given class. Filled squares and triangles represent $\langle C E\rangle$ for two sets of variability classes. Generally softer classes have smaller $\langle C E\rangle$ and harder classes have higher $\langle C E\rangle$.

It is pertinent to ask what this sequence is good for? In Chakrabarti et al. (2004) and Chakrabarti et al. (2005), it was reported that although many observations were made of GRS 1915+105, only a few cases, direct transitions were observed. In particular, using Indian X-ray Astronomy Experiment (IXAE) they showed direct evidences of $\kappa \rightarrow \rho(\mathrm{VIII} \rightarrow \mathrm{IX}), \chi \rightarrow \rho(\mathrm{XIII} \rightarrow \mathrm{IX}), \chi \rightarrow \theta(\mathrm{XIII} \rightarrow \mathrm{XII})$ and $\rho \rightarrow \alpha$ $(\mathrm{IX} \rightarrow \mathrm{XI})$ transitions in a matter of hours. In Naik et al. (2002b), IXAE data was used to argue that $\rho$ class variability could have changed to $\chi$ class via $\alpha$ class (i.e., IX $\rightarrow$ XI $\rightarrow$ XII). In Nandi et al. (2001), it was shown that the $\theta$ class (Class XII) is rare and the observed soft-dip is perhaps due to the disappearance of the inner region by magnetic rubber band effect. Accepting that class XII as anomalous, we find from Fig. 4 that the observed transitions reported in Chakrabarti et al. (2004, $2005)$ and Naik et al, 2002b are 'naturally' explained. For instance, $\chi \rightarrow \rho(\theta$ and $\beta$ being anomalous and intermediate $\alpha$ has been reported by Naik et al. 2002b), $\rho \rightarrow \alpha$ ( $\beta$ being anomalous) and $\kappa \rightarrow \rho$, are expected from our analysis. Similarly, we can claim that there should not be any transitions such as I $\rightarrow$ IX; III $\rightarrow$ VII; for example. We believe that if we carry out spectro-photometry of GRS $1915+105$ continuously, then we may be able to catch the transition from one type to another more often and verify if the sequences we mentioned here need further refining (Pal et al. 2011a).

\section{Outburst Sources GX339-4 and XTE 1550-564}

It has been recently suggested (Chakrabarti, Debnath, Nandi \& Pal, 2008; Chakrabarti, Dutta \& Pal, 2009) that the outburst sources such as GRO 1655-40 and XTE J1550-564 exhibit very strong time variation of the spectral and timing properties from the beginning to the end of the outburst. The evolution of the spectral states can be fitted well using two component advective flow model (Dutta \& Chakrabarti, 2009). They usually start and end with hard states and 

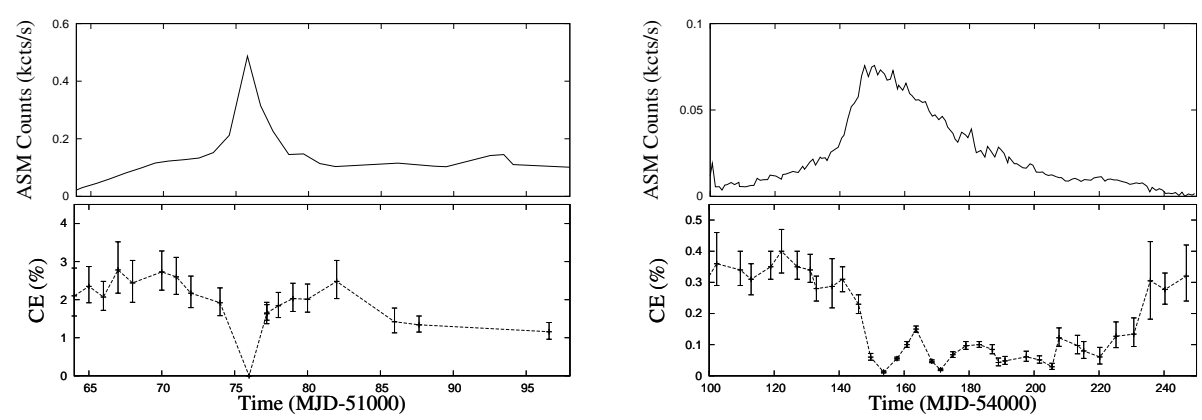

Fig. 5. Evolution of the Comptonization efficiencies in the outburst sources (a) XTE J1550-564 and (b) GX 339-4 respectively. In both the cases CE is higher at the beginning and end of the outbursts, indicated that the Compton cloud geometry was bigger than when the object went into soft and intermediate states.

in between passed through a soft and intermediate states. What about the evolution of the geometry of the flow? Chakrabarti, Debnath, Nandi \& Pal, (2008) and Chakrabarti, Dutta \& Pal (2009) already pointed out that the Compton cloud starts big and becomes smaller at the rising phase of the outburst. In Fig. 5(a-b), we show the results of the evolution of CE in two outbursts, namely XTE 1550-564 (during its 1998 outburst) and GX 339-4 (during its 2007 outburst). We also superpose the light curves. We readily note that the outburst started with a large $\mathrm{CE}$, became smallest at the peak of the outburst and finally became large again (Pal \& Chakrabarti, 2011, submitted).

\section{Gamma Ray Bursts}

The time evolution of GRBs is extremely rapid and it would have been very instructive to compute the evolution of the geometry in this short time. Unfortunately, only a few GRB spectra in their early few seconds showed evidences of black body and power law components (Ghirlanda et al. 2007). Recently, Izzo et al. (2011) have indicated that that the enigmatic GRB 090618 (Rao et al. 2011) also exhibits the power-law and black body components (Ruffini et al. 2011). While Rao et al. (2011) show that the first component has an unusually long time lag $(\sim 7 \mathrm{~s})$, based on which Ruffini et al. (2011) presented the possibility of a multiple component, Izzo et al. (2011) suggests that the pre-GRB emission episode could be the signature of the formation of the black hole itself. In Fig. 6a, we show the dynamical spectrum of GRB 090618 and Fig. 6b shows the Comptonizing efficiency for the black body and power-law fit of the first episode. Note that in the first 50 seconds (Episode 1) the $\mathrm{CE}$ is large. Thus, if the power photons do arise out of Comptonization of some of the injected seed photons, its size was large. As time passes by, the source of the black body component grew and possible overshoot the Compton cloud itself. This is because, the fit became increasingly difficult towards the end of the first Episode. The growth of the black body component has been discussed in Izzo et al. (2011). 

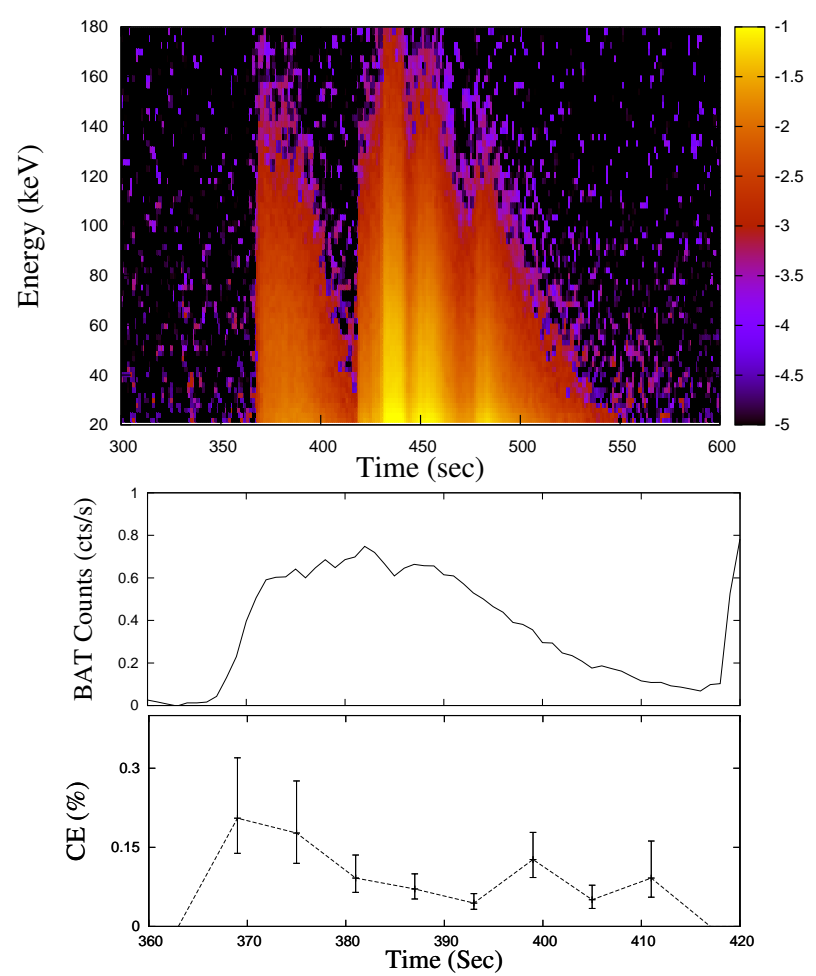

Fig. 6. Dynamical evolution of the (a) spectrum and (b) CE of the GRB 090618. CE is large at the beginning, but has started becoming smaller towards the end of the first episode. The sharp rise and slower decay of the spectrum indicates that the spectrum started hard but becomes softer rapidly.

\section{Concluding remarks}

In this paper, we discussed a new way to study the evolution of the geometry of a system emitting a radiation which could be described as a sum of black body and a power law. The ratio between the photon numbers in the power-law and the black body components gives the so-called Comptonization efficiency (CE) which is a measure of the fraction of the injected seed photons intercepted by the Compton cloud. A larger CE means a larger Compton cloud, i.e., a harder state. We took a few enigmatic sources: a black hole candidate GRS1915+105 which exhibited 16 types of light curves. The CE obtained are aligned perfectly in the same sequence in which the variability class transition occurs, at least for the cases whenever the data is available. We find that CE roughly varies from $0.8 \%$ for harder classes to about $0.03 \%$ for softer classes. We also analysed the cases of two outbursts and showed that the CE was very large in the beginning and the end of the outbursts while it is very small in between when the spectrum was very soft. In the case of GRBs, only early stages are reported to have black body plus power-law components, but the interpretations vary. Assuming the power-law is generated from a black body, 
we find that the variation of CE points to an expanding black body source possibly engulfing a Compton cloud. These aspects would be studied in more detail and the results would be published elsewhere (Pal and Chakrabarti, 2011).

The work of P. S. Pal is supported by CSIR project.

\section{References}

1. Belloni, T., Klein-Wolt, M., Mendez, M., van der Klis, M. \& van Paradijs, J., 2000, A\&A 355, 271

2. Chakrabarti, S. K., Debnath, D., Nandi, A. \& Pal, P.S., 2008, A\&A, 489, 41

3. Chakrabarti, S. K., Dutta, B. G., Pal, P. S., 2009, MNRAS, 394, 1463

4. Chakrabarti, S. K. \& Manickam, S.G. 2000, ApJ, 531, L41

5. Chakrabarti, S. K. \& Nandi, A. 2000, IJP, 75B(1), 1

6. Chakrabarti, S. K., Nandi, A., Chatterjee, A. K., Choudhury, A. K. \& Chatterjee, U., 2005, A\&A, 431, 825

7. Chakrabarti, S. K., Nandi, A., Choudhury, A. \& Chatterjee, U., 2004, ApJ, 607, 406

8. Chakrabarti, S. K. \& Titarchuk, L. G., 1995, ApJ 455, 623

9. Coppi,P. \& Zdziarski, A. A., 1992, ApJ, 398, 37

10. Dutta, B.G. \& Chakrabarti, S.K., 2010, MNRAS, 404, 2136

11. Ghirlanda, G,, Z. Bosnjak, G. Ghisellini, F. Tavecchio, C. Firmani MNRAS 2007, 379, 73

12. Greiner, J., Morgan, E. H. \& Remillard, R. A., 1996, ApJ, 473, 107

13. Izzo, I. et al., 2011, A\& A (Submitted)

14. Klein-Wolt, M., et. al. 2002, MNRAS 331, 745

15. Kobayashi, Y., Kubota, A., Nakazawa, K., Takahashi, T. \& Makishima, K. 2003, PASJ, 55,

16. F. Haardt \& L. Maraschi, 1993, ApJ 413, 507273

17. Migliari, S. \& Belloni, T. 2003, A\&A 404, 283

18. Mirabel, I. F., Rodriguez, L. F. 1994, Nat 371, 46

19. Morgan, E. H., Remillard, R. A. \& Greiner, J. 1997, ApJ 482, 993

20. Muno M. P., Morgan, E. H. \& Remillard, R. A., 1999, ApJ 527, 321

21. Naik, S. \& Rao, A. R. 2000, A\&A 362, 691

22. Naik, S., Rao, A. R., \& Chakrabarti, S. K., 2002a, JApA 23, 213

23. Naik, S., Agrawal, P.C., Rao, A.R. \& Paul, B., 2002b, MNRAS 330, 487

24. Nandi, A., Manickam, S. G. \& Chakrabarti, S. K., 2000, IJP 74B, 331

25. Pal, P.S., Chakrabarti, S.K. \& Nandi, A., 2011a, IJMPD (in press)

26. Pal, P.S., Chakrabarti, S.K. \& Nandi, A., 2011b, A\&A (Submitted)

27. Rao, A.R., Yadav, J.S., and B. Paul, 2000, ApJ, 544, 443

28. Rao, A. R., Naik, S., Vadawale, S. V. \& Chakrabarti, S.K., 2000, A\&A 360, 25

29. Rao, A. R., et al. 2011, ApJ, 728, 42

30. Ruffini, R., Chakrabarti, S.K. \& Izzo, L., 2011, AdSR (Submitted)

31. Shakura, N. I. \& Sunyaev, R. A. 1973, A\&A 24, 337

32. Sunyaev, R. A. \& Titarchuk, L. G., 1980, ApJ 86, 121

33. Vadawale, S. V., Rao, A. R. \& Naik, S., et al., 2003, ApJ 597, 1023

34. Yadav, J.S. et al., 1999, ApJ, 517, 935 